\title{
The CTLA4 region as a general autoimmunity factor: An extended pedigree provides evidence for synergy with the HLA locus in the etiology of type 1 diabetes mellitus, Hashimoto's thyroiditis and Graves' disease
}

\author{
Elisabet Einarsdottir*, ${ }^{*}, 7$, Ingegerd Söderström ${ }^{2,7}$, Anna Löfgren-Burström ${ }^{1,3}$, \\ Susann Haraldsson ${ }^{1,3}$, Sofie Nilsson-Ardnor ${ }^{1,3}$, Carlos Penha-Goncalves ${ }^{1,6}$, Lisbet Lind ${ }^{3,4}$, \\ Gösta Holmgren ${ }^{3,4}$, Monica Holmberg ${ }^{5}$, Kjell Asplund ${ }^{2}$ and Dan Holmberg ${ }^{*, 1,3}$ \\ ${ }^{1}$ Umeå Centre for Molecular Medicine, Umeå University, Umea, Sweden; ${ }^{2}$ Department of Medicine, Umeå University, \\ Umea, Sweden; ${ }^{3}$ Unit for Genome Research, Umea University, Umea, Sweden; ${ }^{4}$ Department of Medical Biosciences, \\ Umea University, Umea, Sweden; ${ }^{5}$ Department of Microbiology, Umea University, Umea, Sweden; ${ }^{6}$ Gulbenkian \\ Institute for Science, Oeiras, Portugal
}

We have identified a large family in the northern part of Sweden with multiple cases of autoimmune diseases, namely type 1 diabetes (T1D), Graves' disease (GD) and Hashimoto's thyroiditis (HT). The family members affected by any of these diseases share a region of $2.4 \mathrm{Mb}$ that comprises among others the CTLA4 gene. We determined that all affected members of the family shared the HLA susceptibility haplotype (DR4-DQA1*0301-DQB1*0302). Analysis of genetic interaction conditioning for HLA haplotype provided strong evidence that the critical region which includes the CTLA4 gene acts together with the HLA locus on the etiology of disease (lodscore $4.20(\theta=0.0)$. The study of this family allowed us to:

(1) reinforce a number of reports on linkage and association of the CTLA4 region to T1D and AITD;

(2) demonstrate that a single haplotypic variant in this region constitutes an etiological factor to disease susceptibility in T1D, GD and HT; (3) reveal a strong genetic interaction of the CTLA4 and HLA loci in the genetic architecture of autoimmune disease; (4) emphasise the value of large pedigrees drawn from isolated populations as tools to single out the effect of individual loci in the etiology of complex diseases. European Journal of Human Genetics (2003) 11, 81 -84. doi:10.1038/sj.ejhg.5200903

Keywords: type 1 diabetes mellitus; autoimmune thyroid disease; CTLA4; HLA; linkage

\section{Introduction}

Type 1 diabetes mellitus, and autoimmune thyroid disease (AITD) (Graves' disease and Hashimoto's thyroiditis) are autoimmune disorders with a complex genetic control that are believed to develop through a process mediated by $\mathrm{T}$ lymphocytes. ${ }^{1}$ In addition to a strong contribution to disease susceptibility by the HLA locus ${ }^{2-4}$ each of these diseases have been reported to be associated and/or linked to the CTLA4 locus, and the co-localised IDDM12 type 1

*Correspondence: D. Holmberg, UCMM, building 6M, Umeå University, 90187 Umeå, Sweden; Tel: 4690785 2702; fax: +46 90785 4400;

E-mail: dan.holmberg@ucmm.umu.se

${ }^{7}$ These two authors have contributed equally to this paper

Received 2 April 2002; revised 1 August 2002; accepted 26 August 2002 diabetes mellitus susceptibility locus on chromosome $2 q 33 .^{5-8}$ Whether the same allele, different alleles in the same locus, or different loci in strong linkage disequilibrium mediate this increase in risk, remains unclear. To address this question, it is informative to study large pedigrees, in which the diseases are concurrent and that contain members that are affected with one of these autoimmune diseases.

\section{Materials and methods}

The identification of a large 5-generation family from northern Sweden with multiple members affected by type 1 diabetes mellitus or AITD (Figure 1) allowed us to investigate the common etiology hypothesis. Family members were coded with a binary phenotype representing the 


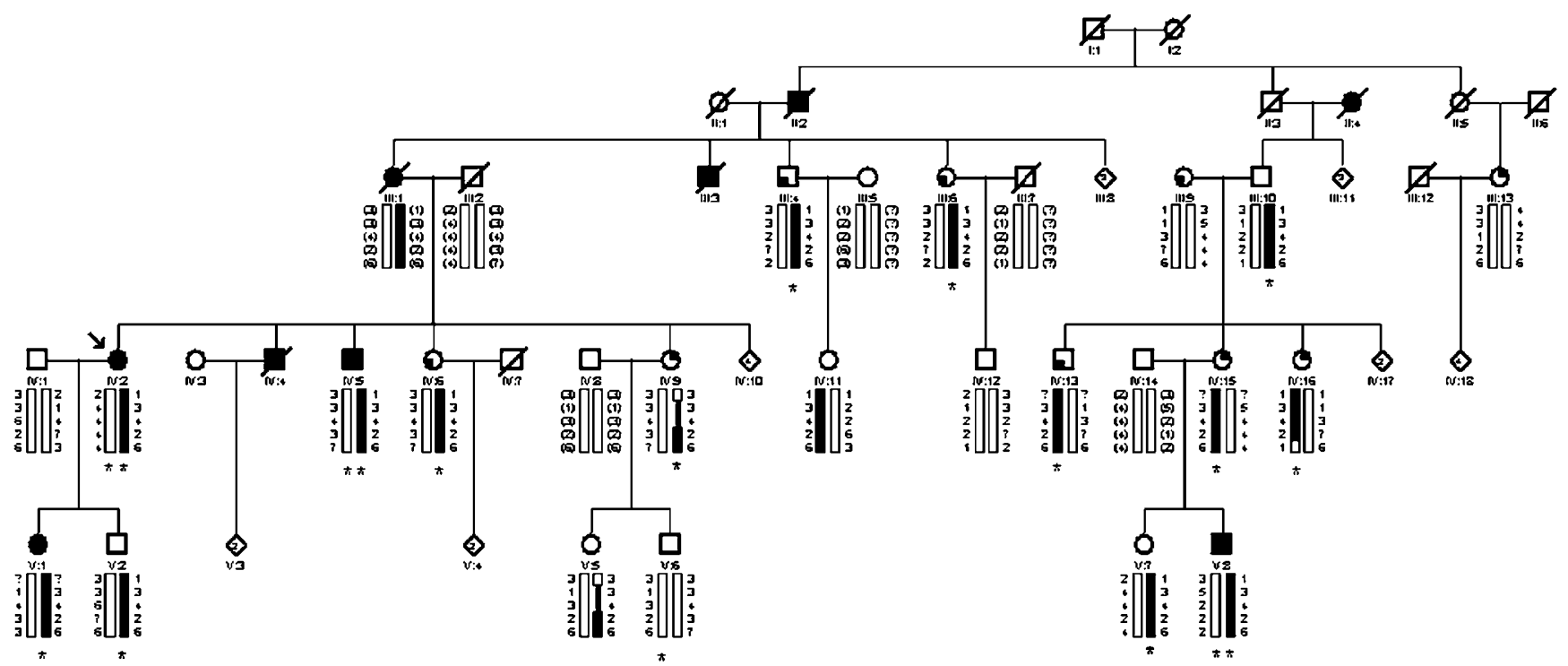

Figure 1 Pedigree of a five-generation family from northern Sweden. Cases of type 1 diabetes mellitus (filled symbols), GD (filled upper right quadrant), or HT (filled lower left quadrant). Alleles corresponding to markers D2S2214, D2S307D2S72, D2S2189 and D2S2237 are shown from top to bottom. Recombination events in individuals IV:9 and IV:16 restrict the disease critical region to a $2.4 \mathrm{Mb}$ interval flanked by D2S2214 and D2S1384. A star beneath the individual indicates presence of the disease associated HLA haplotype. Arrow indicates proband. Only children to patients with the disease haplotype are shown.

presence or absence of autoimmune disease; blood from 72 family members was collected and analysed. Diagnosis of type 1 diabetes mellitus was according to the criteria of the National Diabetes Data Group. ${ }^{9}$ Within the family there are four patients with type 1 diabetes mellitus, seven patients with HT and four with GD. None of the patients were affected with both type 1 diabetes mellitus and AITD. Age of onset was $39.0 \pm 4.5$ years (mean \pm SD) for type 1 diabetes mellitus, $43.6 \pm 9.0$ for GD and $49.8 \pm 9.4$ for HT. Sera from the patients were tested for anti-GAD autoantibodies, but were found to be negative. It should be noted, however, that all patients were tested several years after onset of disease. The study was carried out with the approval of the regional ethical review board and after informed consent of the individuals included in the study.

Genomic DNA was prepared from leucocytes using standard methods. ${ }^{10}$ Using a candidate region approach the family members were genotyped for marker loci in the $H L A$ region on chromosome $6 \mathrm{p} 21$ and in the CTLA4 region on chromosome 2q33. Two-point parametric linkage analysis was carried out using a model of dominant inheritance with 90\% penetrance. An alternative disease model was also used assuming autosomal dominant inheritance with an agedependent penetrance. Using this model, however, did not alter the maximum lodscores obtained more than marginally. Although previous epidemiological studies have reported disease prevalence for type 1 diabetes mellitus of $0.4 \%$ and of $1 \%$ for AITD, ${ }^{13,14}$ the familial form of type 1 diabetes mellitus /AITD studied here is likely to be very rare and the disease allele frequency was therefore specified as 0.001 .

\section{Results}

The marker loci used included known polymorphisms in CTLA4 as well as several microsatellite markers in the region (see Figure 2B). Using this approach, a positive maximum two-point lodscore of 0.72 at $\theta=0.25,0.89$ at $\theta=0.30$ and 0.74 at $\theta=0.16$ was obtained over the marker loci $D 2 S 2392$, D2S309, and D2S72, respectively. These data indicated linkage to a susceptibility locus in this region (Figure 2C).

Next, $H L A$-allele determination was performed for all collected subjects. We noted that all of the affected members of the family were found to carry at least one copy of the previously identified, major HLA susceptibility haplotype contributing to type 1 diabetes mellitus in northern Sweden (DR4-DQA1*0301-DQB1*0302). ${ }^{15}$ This haplotype has a high frequency in this population and $23 \%$ of the unaffected family members had one or more copies of it. It is therefore relatively uninformative and, indeed, no significant positive lodscore was obtained when the HLA locus was analysed independently for linkage to the disease (data not shown). In contrast, however, when the family members were analysed for linkage to markers in the CTLA4 region, using the same model parameters as specified for the two point analysis discussed above but conditioning with respect to HLA DR4-DQA1*0301DQB1*0302 haplotype, a maximum lodscore of 2.69 at $\theta=0.0$ was obtained over the D2S2392 marker locus (Figure 2C). Stratifying on the basis of $H L A$ haplotype, all family members not carrying one or more copies of the HLA DR4-DQA1*0301-DQB1*0302 haplotype were set as having an 'unknown' disease status. This method of conditioning 
A

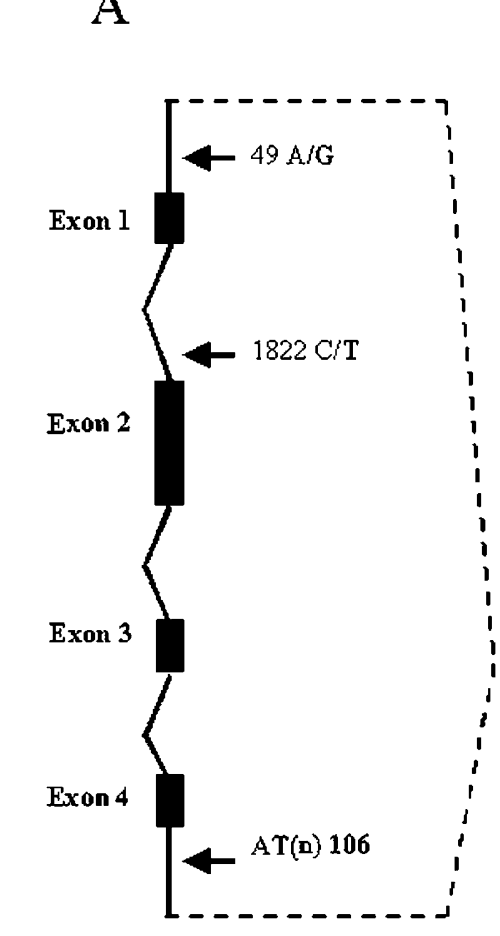

B

C

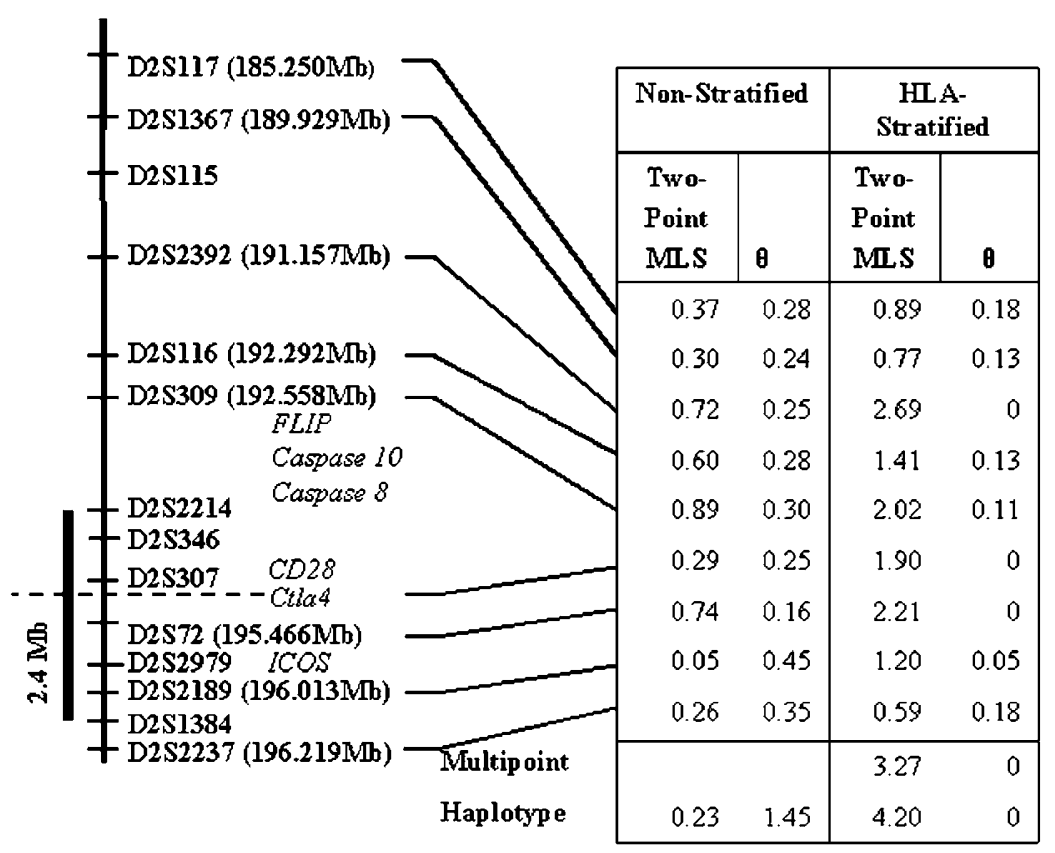

Figure 2 Map of the chromosome 2 q33 region. (A) CTLA4. Polymorhpisms identified in the disease associated allele of CTLA4. Alleles represented in the disease-associated haplotype are highlighted. (B) Critical markers used for linkage analysis and the disease critical region is indicated. Table shows maximum two-point and multipoint lodscore for markers in the 2 33 region.

for HLA haplotype has proven useful in other studies of complex diseases allowing the identification of additional susceptibility loci. ${ }^{16}$

Multipoint analysis using the markers CTLA4(AT)n, D2S72 and D2S297, and using LINKMAP (part of the LINKAGE package ${ }^{11}$ and VITESSE $^{12}$ provided a maximum lodscore of 3.27 close to the marker D2S72 when conditioning for $H L A$.

To identify the disease critical region on chromosome 2q33, affected family members were submitted to haplotype analysis. Minimising the number of recombination events, a $2.4 \mathrm{Mb}$ region restricted by the markers D2S2214 and D2S1384 could be defined as the region of interest (Figure $2 \mathrm{~B})$. Interestingly, one of the recombinations identified allows us to exclude a cluster of apoptosis-related genes previously considered as possible candidates for the IDDM12 susceptibility locus including CASP8, CASP1O and FLIP. Using additional marker loci previously identified in this region we constructed extended haplotypes between these markers and used these for reanalysis of linkage. Using the model parameters specified above and conditioning on HLA haplotype, this approach provided a maximum two-point lodscore of 4.20 at $\theta=0.0$. Without conditioning for $H L A$, the corresponding maximum lodscore obtained was 1.45 at $\theta=0.23$. Together these analyses provide strong evidence in favour of linkage.

Scrutiny of the disease critical region revealed the presence of 46 known or predicted genes including CTLA4,
CD28 and ICOS. Particular interest has been paid to CTLA4 as a candidate for mediating susceptibility to autoimmune disease due to its role as a regulator of $\mathrm{T}$ lymphocyte activity. ${ }^{17,18}$ To search for etiological mutations in the CTLA4 gene, we sequenced genomic DNA from affected and nonaffected members of the family (Figure 2A). The diseaseassociated haplotype identified in the family, was found to carry a combination of three previously identified disease-associated alleles (the $G$ allele in the $A / G$ polymorphism at position 49 in exon 1 , the $\mathrm{T}$ allele in the $\mathrm{C} / \mathrm{T}$ polymorphism in position 1822 in the intron between exon 1 and 2, and the $106 \mathrm{bp}$ allele in the CTLA4 (AT)n polymorphism in the $3^{\prime}$ UT region). ${ }^{5-8,19-22}$

\section{Discussion}

The observed linkage of susceptibility factors for type 1 diabetes mellitus/AITD to HLA and to the CTLA4 region confirms previous reports of linkage/association of type 1 diabetes mellitus, GD and HT to these loci. Moreover, it provides evidence that susceptibility to each of these diseases can be mediated by the same etiologic factor. Thus, it is highly unlikely that more than one allelic form of the same gene or genes, in strong linkage disequilibrium, is mediating susceptibility to each of the different diseases in this family.

Two of the polymorphisms found in the CTLA4 gene, the $49 \mathrm{~A} / \mathrm{G}$ in exon 1 and the AT repeat region located in the $3^{\prime}$ 
UT region, have been suggested to contribute functionally to disease. ${ }^{5,6,23}$ We find it unlikely, however, that these polymorphisms would constitute the etiological mutations explaining the dominant inheritance pattern and high penetrance observed in this family. On the other hand, accumulation of risk factors, that each contribute only a limited risk increase, as well as extended SNP haplotypes affecting gene expression have previously been suggested to contribute susceptibility to other complex diseases. ${ }^{24}$ Additional polymorphisms of this type could, in principle, be present also in several different candidate genes in strong linkage disequilibrium. Recent findings in the NOD mouse, where the susceptibility loci Idd5 and Idd9 have been shown to be constituted by several, closely linked susceptibility genes, illustrate such a situation. ${ }^{25,26}$ Thus, susceptibility factors in familial forms of complex genetic diseases such as type 1 diabetes mellitus and AITD may be constituted by the combined effect of minor risk factors accumulating in a risk haplotype of the kind identified here. It should be noted, however, that additional genetic factors located in the disease critical region have not been excluded and further analysis of additional candidate genes in the region are ongoing to address this possibility.

\section{Acknowledgements}

We thank the family, which kindly consented to the study and John Todd for critically reading this manuscript. This work was supported by grants from the Swedish Research Council and the Swedish Foundation for Strategic Research. E Einarsdottir was supported by a fellowship from the Swedish Institute and A Löfgren-Burström by a fellowship from the Swedish Foundation for Strategic Research.

\section{References}

1 Eisenbarth GS: Type I diabetes mellitus. A chronic autoimmune disease. N Engl J Med 1986; 314: 1360-1368.

2 Cudworth AG, Woodrow JC: Evidence for HL-A-linked genes in 'juvenile' diabetes mellitus. Br Med J 1975; 3: 133-135.

3 Yanagawa T, Mangklabruks A, Chang YB et al: Human histocompatibility leukocyte antigen-DQA ${ }^{*} 0501$ allele associated with genetic susceptibility to Graves' disease in a Caucasian population. J Clin Endocrinol Metab 1993; 76: 1569-1574.

4 Barlow AB, Wheatcroft N, Watson P, Weetman AP: Association of HLA-DQA1*0501 with Graves' disease in English Caucasian men and women. Clin Endocrinol (Oxf) 1996; 44: 73-77.

5 Yanagawa T, Hidaka Y, Guimaraes V, Soliman M, DeGroot LJ: CTLA-4 gene polymorphism associated with Graves' disease in a Caucasian population. J Clin Endocrinol Metab 1995; 80: 41-45.

6 Nistico L, Buzzetti R, Pritchard LE, Van der Auwera B, Giovannini $\mathrm{C}$, Bosi E et al: The CTLA-4 gene region of chromosome $2 \mathrm{q} 33$ is linked to, and associated with, type 1 diabetes. Belgian Diabetes Registry. Hum Mol Genet 1996; 5: 1075 - 1080.

7 Kotsa K, Watson PF, Weetman AP: A CTLA-4 gene polymorphism is associated with both Graves disease and autoimmune hypothyroidism. Clin Endocrinol (Oxf) 1997; 46: 551- 554.

8 Awata T, Kurihara S, Iitaka M et al: Association of CTLA-4 gene AG polymorphism (IDDM12 locus) with acute-onset and insulindepleted IDDM as well as autoimmune thyroid disease (Graves' disease and Hashimoto's thyroiditis) in the Japanese population. Diabetes 1998; 47: 128-129.
9 National Diabetes Data Group: Classification and diagnosis of diabetes mellitus and other categories of glucose intolerance. Diabetes 1979; 28: 1039-1057.

10 Miller SA, Dykes DD, Polesky HF: A simple salting out procedure for extracting DNA from human nucleated cells. Nucleic Acids Res 1988; 16: 1215

11 Terwilliger JD, Ott J: Handbook of Human Genetic Linkage. Baltimore, 1994.

12 O'Connell JD, Weeks DE: The VITESSE algorithm for rapid exact multilocus linkage analysis via genotype set-recoding and fuzzy inheritance. Nat Genet 1995; 11: $402-408$.

13 Dahlquist G, Blom L, Tuvemo T, Nystrom L, Sandstrom A, Wall S: The Swedish childhood diabetes study-results from a nine year case register and a one year case-referent study indicating that type 1 (insulin-dependent) diabetes mellitus is associated with both type 2 (non-insulin-dependent) diabetes mellitus and autoimmune disorders. Diabetologia 1989; 32: 2-6.

14 Vanderpump MP, Tunbridge WM, French JM et al: The incidence of thyroid disorders in the community: a twenty-year follow-up of the Whickham Survey. Clin Endocrinol (Oxf) 1995; 43: 55-68.

15 Kockum I, Wassmuth R, Holmberg E, Michelsen B, Lernmark A: HLA-DQ primarily confers protection and HLA-DR susceptibility in type I (insulin-dependent) diabetes studied in populationbased affected families and controls. Am J Hum Genet 1993; 53 $150-167$

16 King AL, Yiannakou JY, Brett PM, Curtis D, Morris MA, Dearlove AM et al: A genome-wide family-based linkage study of coeliac disease. Ann Hum Genet 2000; 64: 479-490.

17 Walunas TL, Lenschow DJ, Bakker CY et al: CTLA-4 can function as a negative regulator of $\mathrm{T}$ cell activation. Immunity 1994; 1 : $405-413$.

18 Krummel MF, Allison JP: CD28 and CTLA-4 have opposing effects on the response of T cells to stimulation. J Exp Med 1995; 182: $459-465$.

19 Donner H, Braun J, Seidl C et al: Codon 17 polymorphism of the cytotoxic T lymphocyte antigen 4 gene in Hashimoto's thyroiditis and Addison's disease. J Clin Endocrinol Metab 1997; 82: 41304132.

20 Donner H, Rau H, Walfish PG et al: CTLA4 alanine-17 confers genetic susceptibility to Graves' disease and to type 1 diabetes mellitus. J Clin Endocrinol Metab 1997; 82: 143-146.

21 Braun J, Donner H, Siegmund T, Walfish PG, Usadel KH, Badenhoop K: CTLA-4 promoter variants in patients with Graves' disease and Hashimoto's thyroiditis. Tissue Antigens 1998; 51: $563-566$.

22 Marron MP, Zeidler A, Raffel LJ et al: Genetic and physical mapping of a type 1 diabetes susceptibility gene (IDDM12) to a $100-\mathrm{kb}$ phagemid artificial chromosome clone containing D2S72-CTLA4-D2S105 on chromosome 2q33. Diabetes 2000; 49 : $492-499$.

23 Kouki T, Sawai Y, Gardine CA, Fisfalen ME, Alegre ML, DeGroot LJ: CTLA-4 gene polymorphism at position 49 in exon 1 reduces the inhibitory function of CTLA- 4 and contributes to the pathogenesis of Graves' disease. J Immunol 2000; 165: 6606-6611.

24 Gibson AW, Edberg JC, Wu J, Westendorp RG, Huizinga TW, Kimberly RP: Novel single nucleotide polymorphisms in the distal il-10 promoter affect il-10 production and enhance the risk of systemic lupus erythematosus. I Immunol 2001; 166: 3915 3922.

25 Hill NJ, Lyons PA, Armitage N, Todd JA, Wicker LS, Peterson LB: NOD Idd5 locus controls insulitis and diabetes and overlaps the orthologous CTLA4/IDDM12 and NRAMP1 loci in humans. Diabetes 2000; 49: 1744-1747.

26 Lyons PA, Hancock WW, Denny P et al: The NOD Idd9 genetic interval influences the pathogenicity of insulitis and contains molecular variants of Cd30, Tnfr2, and Cd137. Immunity 2000; 13: $107-115$ 